\title{
Using Minimum Mobile Chargers to Keep Large-scale Wireless Rechargeable Sensor Networks Running Forever
}

\author{
Haipeng Dai*, Xiaobing Wu*, Lijie Xu*, Guihai Chen*†, and Shan Lin ${ }^{\ddagger}$ \\ * State Key Laboratory for Novel Software Technology, Nanjing University, Nanjing, Jiansu 210046, CHINA \\ ${ }^{\dagger}$ Shanghai Key Laboratory of Scalable Computing and Systems, Shanghai Jiao Tong University, Shanghai 200240, CHINA \\ ${ }^{\ddagger}$ Temple University, Philadelphia, USA \\ Emails: \{dhpphd2003,ljxu83\}@gmail.com, \{wuxb, gchen\}@nju.edu.cn, slin@temple.edu
}

\begin{abstract}
Wireless Rechargeable Sensor Networks (WRSNs) can be recharged after deployment for sustainable operations. Recent works propose to use a single mobile charger (MC) traveling through the network fields to recharge every sensor node. These algorithms work well in small scale networks. However, in large scale networks these algorithms do not work efficiently, especially when the amount of energy the MC can provide is limited. To address these challenges, multiple MCs can be used. In this paper, we investigate the minimum MCs problem (MinMCP) for rechargeable sensor networks: how to find the minimum number of energy-constrained MCs and design their recharging routes given a sensor network such that each sensor node in the WRSN maintains continuous work. Our results are three folds. We first prove that for any $\epsilon>0$, there is no $(2-\epsilon)$-approximation algorithm for Distance Constrained Vehicle Routing Problem (DVRP) on a general metric space, which is the best as far as we know. By reducing from DVRP, we prove that MinMCP is NP-hard, and the inapproximability bound for MinMCP is the same as that of DVRP. Then we propose approximation algorithms for this problem. Finally, we conduct simulations to validate the effectiveness of our algorithms.
\end{abstract}

\section{INTRODUCTION}

Recent studies [1]-[6] propose to use a mobile charger (MC), which can transfer energy to any sensor node nearby in Wireless Rechargeable Sensor Networks (WRSNs) [7]. Typically, the MC periodically traverses every node in the network and stays near each node for a short period to recharge it. Research results demonstrate that this approach work well for small scale networks. For large scale wireless sensor networks, a single mobile charger may not be enough. This is because the MC may not carry sufficient energy to recharge every node in a large scale network on a single tour. Therefore, the MC needs to return to the base station after recharging a part of the network. As a result, single $\mathrm{MC}$ recharging algorithms become invalid and continuous working of sensor nodes can no longer be guaranteed.

To recharge a large scale sensor network, it is necessary to use multiple energy constrained mobile chargers. In this work, we investigate the minimum mobile charger problem for wireless sensor networks. That is, finding the minimum number of energy-constrained MCs as well as their routes to recharge a given WRSN such that each sensor node in the
WRSN maintains continuous work. This problem is highly challenging as we should jointly considered the energy constraints of MCs and the time-sensitive charging requirements of sensor nodes when determining the routes of MCs. We prove that this problem is NP-hard. Approximation algorithms are proposed to solve this problem. Authors of [8] also employed multiple energy-constrained MCs. However, their work focuses on only one-dimensional (1-D) sensor networks, and their goal is to maximize the ratio of the amount of payload energy to overhead energy. Our solution is designed for two-dimensional (2-D) sensor networks, and concentrates on an entirely different problem compared with [8]. It can be widely used for existing systems.

In particular, our solution is mainly based on the classical results of Distance Constrained Vehicle Routing Problem (DVRP). Though DVRP seems similar to the classical multiple traveling salesman problem (mTSP) which has been extensively studied, it has not received the same amount of attention and its theoretical analysis is quite limited. In this paper, we also present some meaningful theoretical findings for DVRP.

The contributions of this work are as follows.

- We are the first to consider the minimum mobile chargers problem (MinMCP) in general 2-D WRSNs, i.e., how to find the minimum number of energy-constrained MCs and their recharging routes given a 2-D WRSN, so as to keep the network running forever.

- We prove that for any $\epsilon>0$, there is no $(2-\epsilon)$ approximation algorithm for Distance Constrained Vehicle Routing Problem (DVRP) on a general metric space, which is the best result as far as we know. Based on this result, we prove that MinMCP is NP-hard, and its inapproximability bound is the same as that of DVRP.

- We propose approximation algorithms to address MinMCP. Then we conduct simulations to verify our theoretical findings.

The remainder of the paper is organized as follows. In Section II, we investigate some related works. We present preliminaries and background in Section III. In Section IV we first formulate the problem and investigate its hardness. Then we 
propose approximation algorithms and conduct performance analysis respectively. Experimental results are presented in Section V before we conclude the paper in Section VI.

\section{RELATED WORK}

We briefly review some related works according to whether single or multiple MCs are used. Tong et al. [1] investigated the impact of wireless charging technology on deployment of sensor network and routing where an MC is applied. Shi et al. [2] also employed an MC to charge sensor nodes, where the aggregate charging time and travel time is expected to be minimized. A more practical scheme considering routing and charging jointly was reported in [3]. It aims to maximize the network lifetime under practical constraints such as dynamic communication environment. In [9], an MC is used to serve both as a charger and a data collector. Different from [1] [3], [9] which assume that the MC employed has unbounded energy, [10] tried to maximize the number of sensor nodes to be charged by using a single MC with limited energy. Zhang et al. [8] proposed the only scheme employing multiple energyconstrained MCs to collaboratively charge a 1-D WSN. Their goal is to maximize the energy efficiency of charging, which is totally different from ours.

\section{PRoblem Statement}

\section{A. Network Model}

We assume that there is a collection of rechargeable sensor nodes distributed over a 2-D region. A base station (BS) periodically dispatches MCs to charge the sensor nodes. Let $G=(V, E)$ represent the sensor nodes and the BS. Let $v_{B S} \in V$ denote the $\mathrm{BS}$, and $J=V \backslash v_{B S}(|J|=n)$ be the set of sensor nodes. Denote by $w(i, j)$ the time cost of moving from vertex $v_{i}$ to $v_{j}$, which we call the edge weight. We assume that $G$ is complete and the edge weights form a metric space $\mathcal{W}$ (i.e., they are symmetric and satisfy the triangle inequality). This assumption is without loss of generality because an MC can always travel along the shortest path between any two vertices.

Every sensor node has a battery capacity of $E_{\max }$, and needs a minimum energy $E_{\min }$ to be operational. We assume that the energy consumption rate is constant and uniform for all sensor nodes, and is denoted by $p_{w}$. This assumption holds for a number of scenarios. For example, Jiang et al. [11] studied wireless power transmission for sensor nodes buried inside concrete. These sensor nodes collect valuable volumetric data related to the health of a structure, and wirelessly transmit data to a data collection receiver directly [12]. Therefore, the energy consumptions of sensor nodes are identical. In [4], a civil structure is instrumented with sensor nodes capable of being charged wirelessly by a mobile helicopter, which also serves as a data collector. Thus hop-by-hop data transmissions are no longer needed, and the energy consumptions can be conserved and balanced on sensor nodes. In addition, RFID sensors in Wireless Identification and Sensing Platform (WISP) [13] [14] typically consume energy at the same rate, and they can be wireless charged by a mobile charger [15].

\section{B. Charging Model}

Suppose that a fully charged MC $k$ starts from the BS and visits every node of a subset of sensor nodes $S_{k} \in J$ exactly once and charges them. MC $k$ spends $\tau_{i}^{k}$ units of time in charging a node $v_{i}$. Denote the tour of MC $k$ by $P_{k}=\left(\pi_{0}, \pi_{1}, \ldots, \pi_{\left|S_{k}\right|}, \pi_{\left|S_{k}\right|+1}\right)$ where $\pi_{0}=\pi_{\left|S_{k}\right|+1}=v_{B S}$ and $\left\{\pi_{i}\right\}_{i=1}^{\left|S_{k}\right|}=S_{k}$. Consequently, the time MC $k$ takes to travel along $P_{k}$ is given by $\tau_{P}^{k}=\Sigma_{i=0}^{\left|S_{k}\right|} w\left(\pi_{i}, \pi_{i+1}\right)$. Further, let $\mathcal{M}$ be the set of tours of all MCs, which means that $P_{k} \in \mathcal{M}$ and $k \in[|\mathcal{M}|]$ for any $\mathrm{MC} k$.

After finishing the charging, $\mathrm{MC} k$ returns to the $\mathrm{BS}$ to be serviced (e.g., replacing or recharging its battery) and gets ready for the next trip. This period is called vacation time, and is denoted as $\tau_{v a c}^{k}$. We demand that $\tau_{v a c}^{k}$ for any MC $k$ should not be smaller than a given constant value $\tau_{v a c}^{c}$, i.e.,

$$
\tau_{\text {vac }}^{k} \geq \tau_{\text {vac }}^{c} \quad(k \in[|\mathcal{M}|])
$$

in order to meet the requirements of most applications. MC $k$ repeats its recharge schedule every period of time $\tau^{k}$, which consists of charging time, travel time and vacation time, i.e.,

$$
\tau^{k}=\tau_{P}^{k}+\tau_{v a c}^{k}+\Sigma_{v_{i} \in S_{k}} \tau_{i}^{k} \quad(k \in[|\mathcal{M}|]) .
$$

Let $U\left(U>p_{w}\right)$ be the energy transfer rate of an MC during charging. To ensure that each sensor node maintain continuous work and the charging cost of an MC is minimized, the following two conditions proposed by [2] should be satisfied.

$$
\begin{gathered}
\tau^{k} \cdot p_{w}=\tau_{i}^{k} \cdot U \quad\left(k \in[|\mathcal{M}|], v_{i} \in S_{k}\right) \\
E_{\text {max }}-\left(\tau^{k}-\tau_{i}^{k}\right) \cdot p_{w} \geq E_{\min } \quad\left(k \in[|\mathcal{M}|], v_{i} \in S_{k}\right) .
\end{gathered}
$$

The first equation indicates that the amount of energy charged to node $v_{i}$ during $\tau_{i}$ must be equal to that consumed by $v_{i}$ in a schedule period, and the second inequality is obtained by considering the lowest energy level of the node.

Note that, on the contrary, the charging scheme in [8] requires all MCs to have uniform schedule periods, which means that the MCs that finish the charging tasks earlier have to wait at BS for the returns of other MCs before starting the next trip. Consequently, to compensate the inefficiency of MCs caused by waiting, more MCs are needed compared with our scheme. Besides, we stress that our scheme can be easily extended to the case where uniform schedule periods for MCs are mandatory. In fact, this case is much simpler to handle than that we consider in this paper.

\section{Energy Consumption Model for MCs}

Suppose that each MC is energy-constrained and has a maximum energy capacity of $B$. Furthermore, denote by $\eta_{C}$ and $\eta_{T}$ the working powers of each $\mathrm{MC}$ for travel and for charging, respectively. For each MC, the movements and the charging process share the same pool of battery energy. Clearly, the overall energy an MC spends in travel and charging should not exceed its maximum battery capacity $B$. That is,

$$
\eta_{T} \cdot \tau_{P}^{k}+\eta_{C} \cdot \Sigma_{v_{i} \in S_{k}} \tau_{i}^{k} \leq B
$$


In most cases, $\eta_{T}$ is much bigger than $\eta_{C}$. But there are exceptions. For instance, the helicopter in [4] which serves as an MC might spend more energy when hovering for charging sensor nodes than that when flying, i.e., $\eta_{C}>\eta_{T}$. We include this case in the theoretical part.

\section{Problem Description}

Definition 3.1: Minimum Mobile Chargers Problem (MinMCP): Given a set of sensor nodes $J$ with parameters $p_{w}$, $E_{\text {max }}$ and $E_{\text {min }}$, the metric space $\mathcal{W}$ including the time cost of an MC to travel between any pair of nodes, find a minimum required number of MCs (with parameters $\eta_{C}, \eta_{T}, U, B$ and $\tau_{\text {vac }}^{c}$ ) originating at the $B S$ and collectively visiting all the sensor nodes to charge them.

Combining Eqs. (1) (2) (3) (4) (5), and the fact that $\tau^{k}, \tau_{i}^{k}>$ 0 , we formulate our problem as follows.

Min

$$
\begin{aligned}
& \text { s.t. } \quad \tau_{P}^{k} \leq\left(\alpha-\tau_{\text {vac }}^{c}\right)-\lambda \cdot \alpha \cdot\left|S_{k}\right| \quad(k \in[|\mathcal{M}|]) \\
& \tau_{P}^{k} \leq \frac{B-\lambda \cdot\left(B+\eta_{C} \cdot \tau_{\text {vac }}^{c}\right) \cdot\left|S_{k}\right|}{\eta_{T}+\lambda \cdot\left(\eta_{C}-\eta_{T}\right) \cdot\left|S_{k}\right|}(k \in[|\mathcal{M}|]) \\
& \cup_{k \in[|\mathcal{M}|]} S_{k}=J ;\left|S_{k}\right|<\frac{1}{\lambda} ; S_{i} \cap S_{j}=\emptyset \\
&(i, j, k \in[|\mathcal{M}|], i \neq j)
\end{aligned}
$$

where $\alpha=\frac{U \cdot\left(E_{\max }-E_{\min }\right)}{p_{w} \cdot\left(U-p_{w}\right)}$ and $\lambda=\frac{p_{w}}{U}$.

Define $f_{1}\left(\left|S_{k}\right|\right)=\left(\alpha-\tau_{v a c}^{c}\right)-\lambda \cdot \alpha \cdot\left|S_{k}\right|$ and $f_{2}\left(\left|S_{k}\right|\right)=$ $\frac{B-\lambda \cdot\left(B+\eta_{C} \cdot \tau_{v a c}^{c}\right) \cdot\left|S_{k}\right|}{\eta_{T}+\lambda \cdot\left(\eta_{C}-\eta_{T}\right) \cdot\left|S_{k}\right|}$. For simplicity, we use $f_{1}\left(\left|S_{k}\right|\right)$ (or $f_{2}\left(\left|S_{k}\right|\right)$ ) to represent the constraint $\tau_{P}^{k} \leq f_{1}\left(\left|S_{k}\right|\right)$ (or $\left.\tau_{P}^{k} \leq f_{2}\left(\left|S_{k}\right|\right)\right)$, if there is no confusion. In addition, we assume that $2 \cdot w\left(v_{B S}, v_{i}\right) \leq f_{1}(1)$ and $2 \cdot w\left(v_{B S}, v_{i}\right) \leq f_{2}(1)$ for any node $v_{i} \in J$, otherwise there is no feasible solution for MinMCP.

\section{SOlving MinMCP: Theoretical Results And APPROXIMATION ALGORITHMS}

In this section, we find that MinMCP is difficult to tackle directly after examining the hardness of MinMCP. Then we resort to solving its relaxed version MinMCP-R. Finally we come up with an approximation algorithm for MinMCP based on the solution to MinMCP-R.

\section{A. Hardness of MinMCP}

First, we review the following NP-complete problem.

Definition 4.1: Distance Constrained Vehicle Routing Problem (DVRP) [16]: Given a set of vertices in a metric space, a specified depot, and a distance bound D, find a minimum cardinality set of tours originating at the depot that covers all vertices, such that each tour has length at most $D$.

Theorem 4.1: For any $\epsilon>0$, there is no $(2-\epsilon)$ approximation algorithm for DVRP unless $P=N P$.

Proof: We reduce from the traveling salesman problem (TSP). It is well known that the decision version of the TSP (where, given a length $L$, the task is to decide whether any tour is shorter than $L$ ) belongs to the class of NP-hard problems.

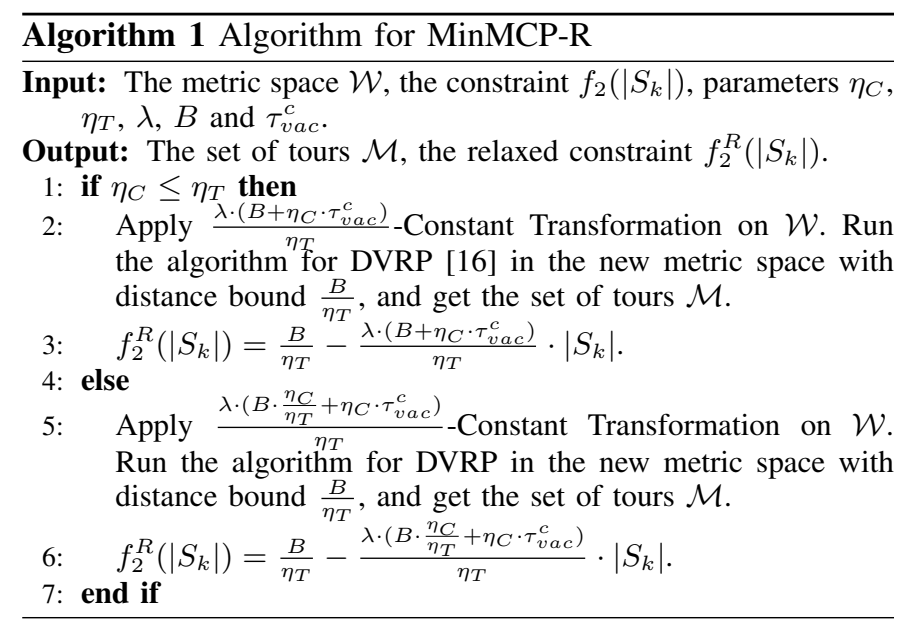

Given an instance of the TSP decision problem with a given length $L$, we construct an instance of DVRP by setting the distance bound $D=L$. Apparently, for any $\epsilon>0$, if there exists a $(2-\epsilon)$-approximation algorithm to DVRP, we can use it to obtain a single tour traversing all nodes given that the answer to the decision problem of the TSP is yes. In other words, we can solve the decision problem of the TSP in polynomial time by utilizing the approximation solution. This is impossible unless $P=N P$.

We emphasize that this obtained inapproximability bound is the best result for DVRP on a general metric space, which improves the best existing bound $3 / 2$ [17].

Theorem 4.2: MinMCP is NP-hard, and for any $\epsilon>0$, there is no $(2-\epsilon)$-approximation algorithm for MinMCP unless $P=N P$.

Proof: Suppose $B$ is sufficiently large and $U \gg p_{w}$, thus the constraint $f_{2}\left(\left|S_{k}\right|\right)$ can be relaxed and $f_{1}\left(\left|S_{k}\right|\right)$ can be rewritten as $\tau_{P}^{k} \leq\left(\alpha-\tau_{v a c}^{c}\right)$. Hence, for any instance of DVRP, we can construct in polynomial time an instance of MinMCP for this special case. Since DVRP is NP-complete and thus is NP-hard, we conclude that MinMCP is also NPhard. Furthermore, as MinMCP is at least as hard as DVRP, according to Theorem 4.1, for any $\epsilon>0$, there is no $(2-\epsilon)$ approximation solution to MinMCP unless $P=N P$.

\section{B. Roadmap of Our Solution}

Our roadmap to solve MinMCP is as follows. Due to the difficulty of tackling MinMCP directly, we first consider its relaxed version where the linear constraint $f_{1}\left(\left|S_{k}\right|\right)$ is removed, which we call MinMCP-R.

For MinMCP-R, we first relax the nonlinear constraint $f_{2}\left(\left|S_{k}\right|\right)$ into a linear one, and then reduce the problem to DVRP by applying a simple transformation. This approach allows us to propose an approximation algorithm.

Based on the solution to MinMCP-R, we can construct a feasible solution to MinMCP by taking into account the constraint $f_{1}\left(\left|S_{k}\right|\right)$. An approximation algorithm is also provided in this case.

\section{Approximation Algorithm for MinMCP-R}

First of all, we define $\gamma$-Constant Transformation in metric space as follows. 
Definition 4.2: $\gamma$-Constant Transformation: Given a metric space $\mathcal{W}$ and a constant $\gamma$, the $\gamma$-Constant Transformation for $\mathcal{W}$ is to revise the weight $w(i, j)$ between any pair of vertices $i$ and $j$ as $w(i, j)+\gamma$, and $w\left(i, v_{B S}\right)$ between any vertex $v_{i}$ and the $B S$ as $w\left(i, v_{B S}\right)+\gamma / 2$.

We denote by $\Delta$ the maximum distance of any node from the BS, and $\Delta(\gamma)$ the according one after the $\gamma$-Constant Transformation. It is easy to verify the following lemma.

Lemma 4.1: The metric space after a $\gamma$-Constant Transformation is still metric.

We propose our algorithm for MinMCP-R in Algorithm 1 by applying $\gamma$-Constant Transformation. One of the outputs of the algorithm is the relaxed constraint of $f_{2}\left(\left|S_{k}\right|\right)$, i.e., $f_{2}^{R}\left(\left|S_{k}\right|\right)$, a useful element in later sections.

\section{Analysis of the Approximation Algorithm for MinMCP-R}

We give performance bounds of Algorithm 1 for three cases respectively, i.e., $\eta_{C}=\eta_{T}, \eta_{C}<\eta_{T}$ and $\eta_{C}>\eta_{T}$.

Theorem 4.3: Given that $\eta_{C}=\eta_{T}$, Algorithm 1 for MinMCP-R achieves $6 \cdot\left(\left\lceil\log _{2} \frac{B / \eta_{T}}{B / \eta_{T}-2 \Delta\left(\frac{\lambda \cdot\left(B+\eta_{C} \cdot \tau_{v a c}^{c}\right)}{\eta_{T}}\right)+2}\right\rceil+\right.$ 1)-approximation.

Proof: Given $\eta_{C}=\eta_{T}$, the constraint $f_{2}\left(\left|S_{k}\right|\right)$ can be simplified as:

$$
\tau_{P}^{k} \leq \frac{B}{\eta_{T}}-\frac{\lambda \cdot\left(B+\eta_{C} \cdot \tau_{v a c}^{c}\right)}{\eta_{T}} \cdot\left|S_{k}\right| .
$$

Note that $\tau_{P}^{k}=\Sigma_{i=0}^{\left|S_{k}\right|} w\left(\pi_{i}, \pi_{i+1}\right)$. Moreover, the weight of the same tour in the revised metric space after applying $\frac{\lambda \cdot\left(B+\eta_{C} \cdot \tau_{\text {vac }}^{c}\right)}{\eta_{T}}$-Constant Transformation on $\mathcal{W}$ in Algorithm 1 is given by:

$$
\begin{aligned}
\tau_{P}^{k^{\prime}}= & \left(w\left(v_{B S}, \pi_{1}\right)+\frac{1}{2} \cdot \gamma_{1}\right)+\left(w\left(\pi_{\left|S_{k}\right|}, v_{B S}\right)+\frac{1}{2} \cdot \gamma_{1}\right) \\
& +\Sigma_{i=1}^{\left|S_{k}\right|-1}\left(w\left(\pi_{i}, \pi_{i+1}\right)+\gamma_{1}\right)
\end{aligned}
$$

where $\gamma_{1}=\frac{\lambda \cdot\left(B+\eta_{C} \cdot \tau_{v a c}^{c}\right)}{\eta_{T}}$. Combining Eqs. (8)(9) and following the fact that $\tau_{P}^{k}=\Sigma_{i=0}^{\left|S_{k}\right|} w\left(\pi_{i}, \pi_{i+1}\right)$, we have:

$$
\tau_{P}^{k^{\prime}} \leq \frac{B}{\eta_{T}}
$$

Thus our problem is reduced to DVRP with distance bound $\frac{B}{\eta_{T}}$. According to Theorem 3 in [16], the result follows.

Theorem 4.4: Given that $\eta_{C}<\eta_{T}$, Algorithm 1 for MinMCP-R achieves $6 \cdot\left(2 \cdot \frac{B+\eta_{C} \cdot \tau_{v a c}^{c}}{B \cdot \frac{\eta_{C}}{\eta_{T}}+\eta_{C} \cdot \tau_{v a c}^{c}}+\right.$ 1) $\left(\left\lceil\log _{2} \frac{B / \eta_{T}}{B / \eta_{T}-2 \Delta\left(\frac{\lambda \cdot\left(B+\eta_{C} \cdot \tau_{\text {vac }}^{c}\right)}{\eta_{T}}\right)+2}\right\rceil+1\right)$-approximation.

Proof: We prove this theorem in the Appendix for a better flow of the paper.

Theorem 4.5: Given that $\eta_{C}>\eta_{T}$, Algorithm 1 for MinMCP-R achieves $6 \cdot\left(2 \cdot \frac{B \cdot \frac{\eta_{C}}{\eta_{T}}+\eta_{C} \cdot \tau_{\text {vac }}^{c}}{B+\eta_{C} \cdot \tau_{\text {vac }}^{c}}+\right.$ $1)\left(\left\lceil\log _{2} \frac{B / \eta_{T}}{B / \eta_{T}-2 \Delta\left(\frac{\lambda \cdot\left(B \cdot \frac{\eta_{C}}{\eta_{T}}+\eta_{C} \cdot \tau_{\text {vac }}^{c}\right)}{\eta_{T}}\right)+2}\right\rceil+1\right)$-approximation.

Proof: We prove this theorem in the Appendix for a better flow of the paper.

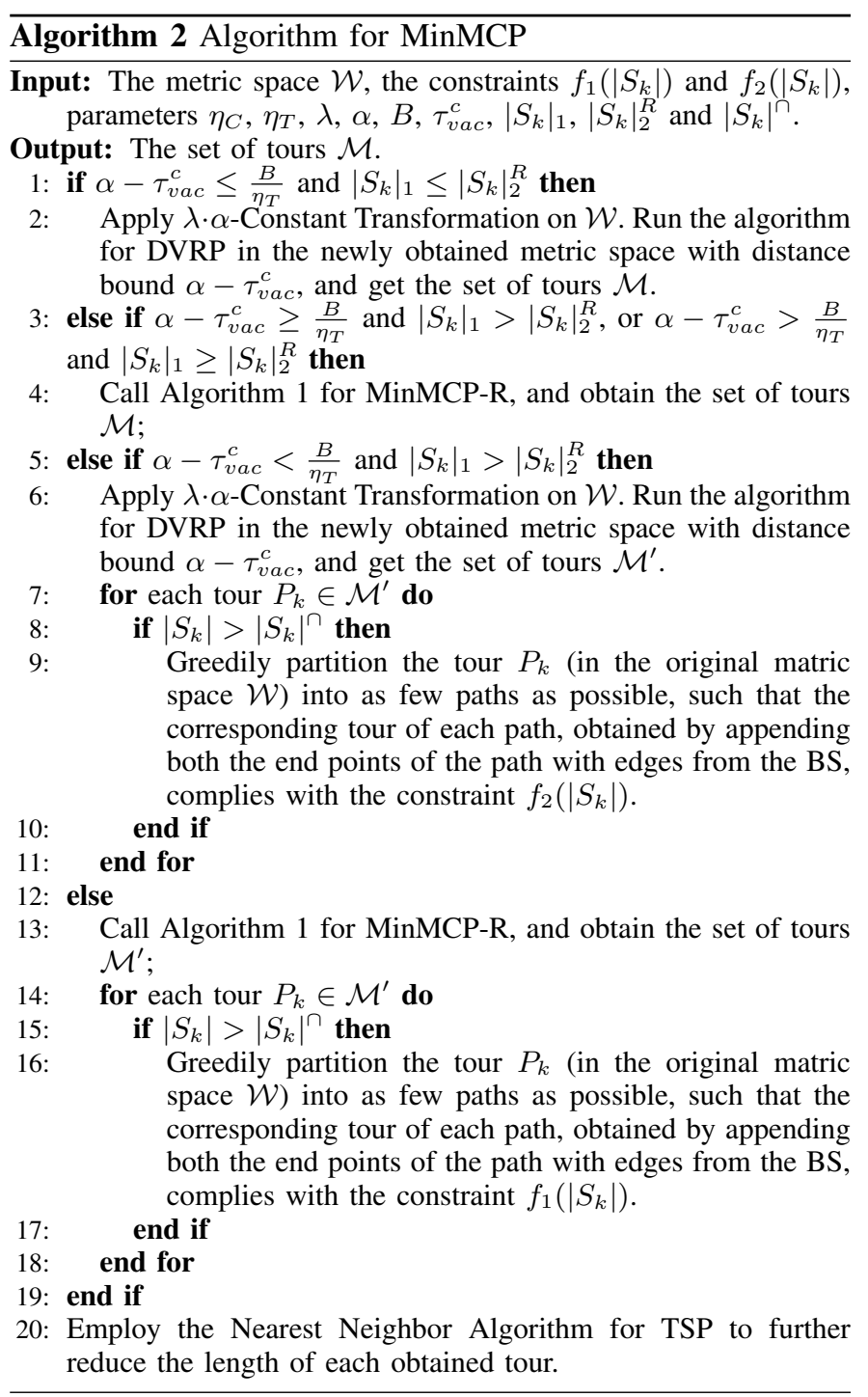

\section{E. Approximation Algorithm for MinMCP}

The core idea of our solution is to use the output of Algorithm 1, $f_{2}^{R}\left(\left|S_{k}\right|\right)$, to substitute the complicated constraint $f_{2}\left(\left|S_{k}\right|\right)$. MinMCP is therefore reformulated as a minimization problem subject to two linear constraints.

Define $\left|S_{k}\right|_{1}=\frac{\alpha-\tau_{v a c}^{c}}{\lambda \cdot \alpha_{B}} \quad\left(f_{1}\left(\left|S_{k}\right|_{1}\right)=0\right)$. If $\eta_{C} \leq \eta_{T}$, define $\left|S_{k}\right|_{2}^{R}=\frac{{ }_{B}}{\lambda \cdot\left(B+\eta_{C} \cdot \tau_{\text {vac }}^{c}\right)}$; otherwise, define $\left|S_{k}\right|_{2}^{R}=$ $\frac{B}{\lambda \cdot\left(B \cdot \frac{\eta_{C}}{\eta_{T}}+\eta_{C} \cdot \tau_{\text {vac }}^{c}\right)}$. Hence, the output of Algorithm $1 f_{2}^{R}\left(\left|S_{k}\right|\right)$ can be rewritten as $f_{2}^{R}\left(\left|S_{k}\right|\right)=\frac{B}{\eta_{T}}-\frac{B}{\eta_{T}} \cdot \frac{1}{\left|S_{k}\right|_{2}^{R}} \cdot\left|S_{k}\right|$. Moreover, define $\left|S_{k}\right|^{\cap}=\frac{\alpha-\tau_{v a c}^{c}-B / \eta_{T}}{\left(\alpha-\tau_{\text {vac }}^{c}\right) /\left|S_{k}\right|_{1}-\left(B / \eta_{T}\right) /\left|S_{k}\right|_{2}^{R}}$.

The pseudo-codes of the algorithm for MinMCP can be found in Algorithm 2. Note that at the final step we employ the Nearest Neighbor Algorithm for TSP to further reduce the length of each obtained tour.

\section{F. Analysis of the Approximation Algorithm for MinMCP}

We present the following two theorems without proofs to save space. Notice that in the scenarios considered in these theorems, the feasible region for $f_{1}\left(\left|S_{k}\right|\right)$ or $f_{2}^{R}\left(\left|S_{k}\right|\right)$ lies entirely inside that of the other constraint, as shown in Fig. 


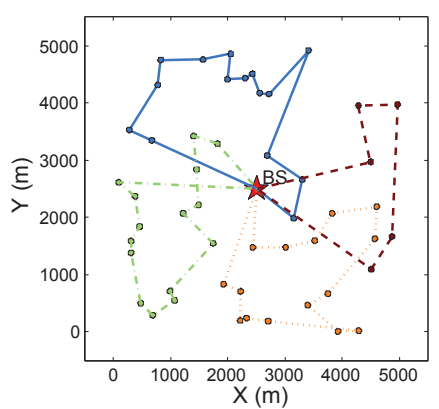

Fig. 1. A Solution to a MinMCP Fig. 2. A Solution to a MinMCP Instance when $B=500 \mathrm{KJ}$

10. Therefore the latter constraint can be safely relaxed and thus MinMCP is subject to only one constraint.

Theorem 4.6: Given that $\alpha-\tau_{\text {vac }}^{c} \leq \frac{B}{\eta_{T}}$ and $\left|S_{k}\right|_{1} \leq\left|S_{k}\right|_{2}^{R}$, Algorithm 2 for MinMCP achieves $6 \cdot\left(\left\lceil\log _{2} \frac{\alpha-\tau_{\text {vac }}^{c}}{\alpha-\tau_{\text {coc }}^{c}-2 \Delta(\lambda \cdot \alpha)+2}\right\rceil+1\right)$-approximation.

Theorem 4.7: Given that $\alpha-\tau_{v a c}^{c} \geq \frac{B}{\eta_{T}}$ and $\left|S_{k}\right|_{1}>\left|S_{k}\right|_{2}^{R}$, or $\alpha-\tau_{\text {vac }}^{c}>\frac{B}{\eta_{T}}$ and $\left|S_{k}\right|_{1} \geq\left|S_{k}\right|_{2}^{R}$, Algorithm 2 for MinMCP achieves $\xi$-approximation, where $\xi$ is the approximation ratio of the corresponding MinMCP-R.

Theorem 4.8: Given that $\alpha-\tau_{v a c}^{c}<\frac{B}{\eta_{T}}$ and $\left|S_{k}\right|_{1}>$ $\left|S_{k}\right|_{2}^{R}$, Algorithm 2 for MinMCP achieves $6 \cdot\left(2 \cdot \frac{\left|S_{k}\right|_{1}}{\left|S_{k}\right|_{2}^{R}}+\right.$ 1) $\left(\left\lceil\log _{2} \frac{\alpha-\tau_{v a c}^{c}}{\alpha-\tau_{c a c}^{c}-2 \Delta(\lambda \cdot \alpha)+2}\right\rceil+1\right)$-approximation.

Proof: We prove this theorem in the Appendix for a better flow of the paper.

As the proof of the following theorem is similar to that of Theorem 4.8, we omit it here to save space.

Theorem 4.9: Given that $\alpha-\tau_{v a c}^{c}>\frac{B}{\eta_{T}}$ and $\left|S_{k}\right|_{1}<$ $\left|S_{k}\right|_{2}^{R}$, Algorithm 2 for MinMCP achieves $2 \cdot\left(\frac{\left|S_{k}\right|_{2}^{R}}{\left|S_{k}\right|_{1}}+1\right) \cdot \xi$ approximation, where $\xi$ is the approximation ratio of the corresponding MinMCP-R.

\section{NUMERICAL RESUltS}

In this section, we present simulation results to verify our theoretical findings. It is noteworthy that in simulations, we don't compare our work to that of [8], which uses multiple MCs to cover a 1-D sensor network, due to the fundamental distinction between them. [8] assumes that MCs can intentionally gather at a rendezvous point to recharge others or to be recharged without energy loss. We think this assumption is somewhat unpractical and therefore do not adopt it.

\section{A. Evaluation Setup}

We randomly distribute 50 sensor nodes in a $5 \mathrm{~km} \times 5 \mathrm{~km}$ 2-D Euclidian region throughout the simulations. Any pair of nodes can reach each other through a direct path. The base station is assumed to be located at $(2500,2500)$ (in m). Unless otherwise specified, we use the following parameter settings as in [2]: the traveling speed of MCs is $V=5 \mathrm{~m} / \mathrm{s} ; p_{w}=$ $200 \mathrm{~mW}, E_{\max }=10.8 \mathrm{~kJ}, E_{\min }=540 \mathrm{~J}, U=5 \mathrm{~W}, \eta_{C}=$ $10 \mathrm{~W}, \eta_{T}=100 \mathrm{~W}, B=200 \mathrm{KJ}, \tau_{v a c}^{c}=1$ hour.

\section{B. Performance Evaluation of MinMCP}

1) Solutions to MinMCP Instances: Fig. 1 illustrates the obtained 4 tours of MCs (marked in different colors and
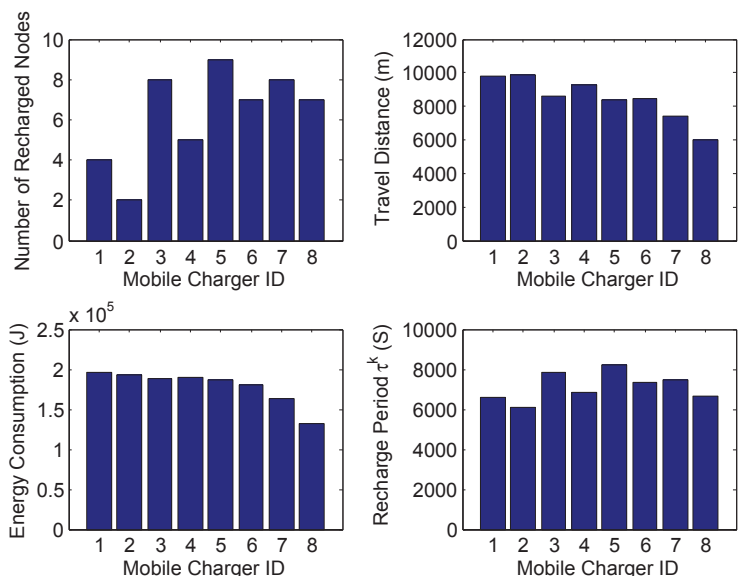

Fig. 3. Critical parameters of each $\mathrm{MC}$ when $B=200 \mathrm{KJ}$

different line styles) for a MinMCP instance, when we set $B=500 \mathrm{KJ}$. However, if $B=200 \mathrm{KJ}$, the number of MCs needed is doubled, as shown in Fig. 2. Note that, for each tour, the Nearest Neighbor Algorithm for TSP is employed to further reduce its length, as is elaborated in Algorithm 2.

For the case in which $B=200 \mathrm{KJ}$, we show the number of charged sensors nodes, total travel distance, energy consumption and recharge period of each $\mathrm{MC}$ in Fig. 3. Note that we set $\tau_{v a c}^{k}=\tau_{v a c}^{c}$ for each MC. It can be seen that energy consumptions are roughly balanced among MCs, and none of them exceeds the maximum battery capacity $B$. Another important observation is that the recharge period $\tau^{k}$ is determined jointly by the number of recharged sensor nodes and the travel distance, rather than solely by one of them. In general, large number of recharged nodes and long travel distance result in large recharge period, such as MC 5. Besides, throughout the recharge period, none of the energy levels of sensor nodes would drop below $E_{\text {min }}$, which can be confirmed by checking the conditions described in Equ. (3) and Inequality (4).

2) Varying $B$ of $M C s$ : In this case, we vary the maximum energy capacity $B$ between $[150 \mathrm{KJ}, 850 \mathrm{KJ}]$ to evaluate its impact on the required number of MCs $|\mathcal{M}|$. Particularly, for each value of $B$, we perform Algorithm 2 under 100 randomly generated topologies, and get the average value of $|\mathcal{M}|$. In addition, the following three situations are evaluated respectively: (i) $\eta_{C}=10 \mathrm{~W}, \eta_{T}=100 \mathrm{~W}$, (ii) $\eta_{C}=100 \mathrm{~W}$, $\eta_{T}=100 \mathrm{~W}$ and (iii) $\eta_{C}=110 \mathrm{~W}, \eta_{T}=100 \mathrm{~W}$. As shown in Fig. 4(a), not surprisingly, it can be seen that the larger $B$ is chosen, the lower $|\mathcal{M}|$ is obtained. Moreover, the required number of MCs $|\mathcal{M}|$ grows as $\eta_{C}$ increases.

Let $\zeta$ be the performance bound given by the theoretical analysis. Though the optimal number of MCs, $\left|\mathcal{M}^{*}\right|$, can be hardly determined, it is certain that $\left|\mathcal{M}^{*}\right| \geq 1$, and therefore the upper bound of the minimum required number of MCs is at least $\zeta$. We plot the ratio of $\zeta /|\mathcal{M}|$ for the three cases in Fig. 4(b). Each point on the curves stands for the average $\zeta /|\mathcal{M}|$ over the 100 randomly generated instances, while the bars above and below the point represent the maximum and minimum values of $\zeta /|\mathcal{M}|$ among the 100 instances, respectively. It can be seen that all the values of $\zeta /|\mathcal{M}|$ for 

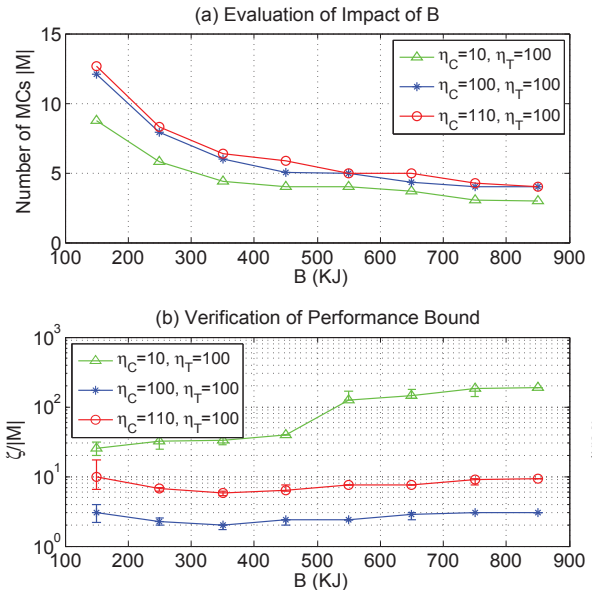

Fig. 4. Evaluation of Impact of $B$ and Verification of Performance Bound
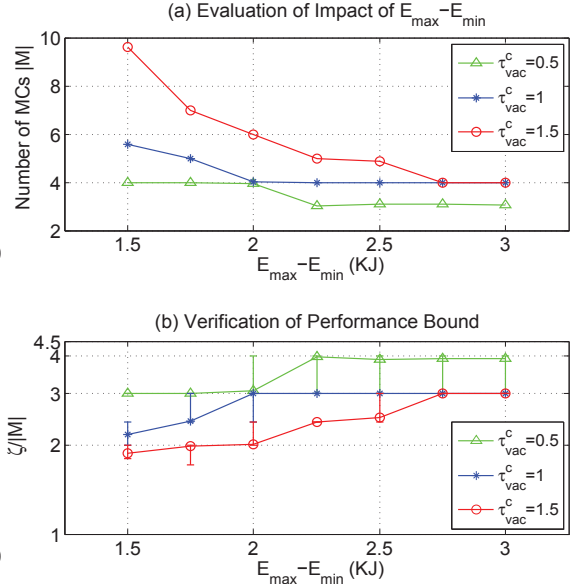

Fig. 5. Evaluation of Impact of $E_{m}$
and Verification of Performance Bound

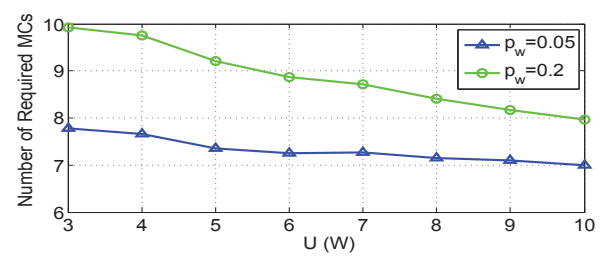

Fig. 6. Evaluation of Impact of $U$

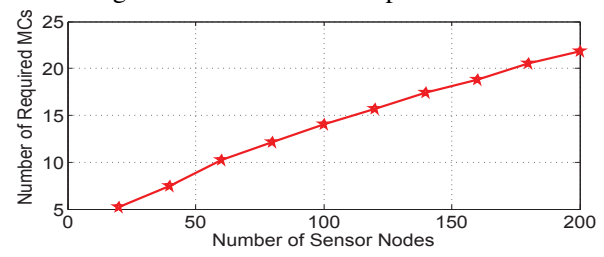

Fig. 7. Evaluation of Impact of Number of Sensor Nodes the three cases, even those minimum values, are greater than 1. This observation corroborates our theoretical findings.

3) Varying $E_{\max }-E_{\min }$ of Sensor Nodes: We proceed to evaluate the impact of $E_{\max }-E_{\min }$ of sensor nodes on the minimum required number of MCs $|\mathcal{M}|$ under the following three cases: (i) $\tau_{v a c}^{c}=0.5$ hour, (ii) $\tau_{v a c}^{c}=1$ hour and (iii) $\tau_{\text {vac }}^{c}=1.5$ hour. Note that we set $B=1000 \mathrm{KJ}$ and $\eta_{C}=\eta_{T}=100 \mathrm{~W}$. Fig. 5(a) shows that $|\mathcal{M}|$ drops with an increasing $E_{\max }-E_{\min }$ or a decreasing $\tau_{v a c}^{c}$. Moreover, the fact that all values of $\zeta /|\mathcal{M}|$ are never less than 1 , as illustrated in Fig. 5(b), is consistent with our theoretical results.

4) Varying $U$ of MCs and Number of Sensor Nodes: We study the impact of energy transfer rate $U$ of an MC on the number of required MCs. Fig. 6 shows that when $U$ rises from $3 W$ to $10 W$, the number of required MCs drops from 9.93 to 7.96 given $p_{w}=0.2 \mathrm{~W}$, and from 7.79 to 6.99 given $p_{w}=0.05 \mathrm{~W}$. Notice that this value is obtained by averaging that of 100 randomly generated topologies. On the other hand, we are also interested in investigating the impact of different number of sensor nodes on the number of MCs. As shown in Fig. 7, the required number of MCs increases steadily from 5.25 to 21.8 while the number of sensor nodes scales from 20 to 200. Accordingly, the average number of recharge nodes of a MC increases significantly (from 3.8 to 9.2 ). This is because the average length of tours is shrunk.

\section{CONCLUSION}

In this paper, we have studied the problem of minimizing the number of energy-constrained MCs to cover a 2-D WSN. We formulate the problem, and show that by applying appropriate transformations, it can be cast into the classical problem-DVRP. We not only prove the NP-hardness and the inapproximability bound of the problem, but also propose approximation algorithms with proven performance bounds.

\section{ACKNOWLEDGMENT}

This work is supported by National 973 project of China under Grant No.2012CB316200, National Natural Science Foundation of China under Grant No.60903179, No.61021062,
No.61073152 and No.61133006, US NSF grants CNS1239108 and CNS-1218718, and Research and Innovation Project for College Graduate Students of Jiangsu Province in 2012 under Grant No.CXZZ12_0056. We would also like to thank Qingyuan Jiang, Chenbin Ji and Xiaojun Zhu for their valuable work.

\section{REFERENCES}

[1] B. Tong, Z. Li, G. Wang, and W. Zhang, "How wireless power charging technology affects sensor network deployment and routing," in ICDCS, 2010.

[2] Y. Shi, L. Xie, Y. T. Hou, and H. D. Sherali, "On renewable sensor networks with wireless energy transfer," in INFOCOM, 2011.

[3] Z. Li, Y. Peng, W. Zhang, and D. Qiao, "J-RoC: a joint routing and charging scheme to prolong sensor network lifetime," in IPSN, 2011.

[4] D. Mascareñas, E. Flynn, M. Todd, G. Park, and C. Farrar, "Wireless sensor technologies for monitoring civil structures," Sound and Vibration, vol. 42, no. 4, pp. 16-21, 2008.

[5] K. Li, H. Luan, and C. Shen, "Qi-ferry: Energy-constrained wireless charging in wireless sensor networks," in Wireless Communications and Networking Conference (WCNC), 2012 IEEE. IEEE, 2012, pp. 25152520.

[6] C. Farrar, G. Park, and M. Todd, "Sensing network paradigms for structural health monitoring," New Developments in Sensing Technology for Structural Health Monitoring, pp. 137-157, 2011.

[7] S. He, J. Chen, F. Jiang, D. K. Y. Yau, G. Xing, and Y. Sun, "Energy provisioning in wireless rechargeable sensor networks," in INFOCOM, 2011.

[8] S. Zhang, J. Wu, and S. Lu, "Collaborative mobile charging for sensor networks," in MASS, 2012.

[9] M. Zhao, J. Li, and Y. Yang, "Joint mobile energy replenishment and data gathering in wireless rechargeable sensor networks," in ITC, 2011.

[10] K. Li, H. Luan, and C. Shen, "Qi-ferry: Energy-constrained wireless charging in wireless sensor networks," in WCNC, 2012.

[11] S. Jiang, "Optimum wireless power transmission for sensors embedded in concrete," 2011.

[12] J. M. Engel, L. Zhao, Z. Fan, J. Chen, and C. Liu, "Smart brick-a low cost, modular wireless sensor for civil structure monitoring," in International Conference on Computing, Communications and Control Technologies (CCCT 2004), Austin, TX USA, 2004.

[13] M. Buettner, R. Prasad, M. Philipose, and D. Wetherall, "Recognizing daily activities with rfid-based sensors," in Proceedings of the 11th international conference on Ubiquitous computing, ser. Ubicomp '09. ACM, 2009, pp. 51-60.

[14] M. Buettner, B. Greenstein, A. Sample, J. Smith, and D. Wetherall, "Revisiting smart dust with rfid sensor networks," in Proceedings of the 7th ACM Workshop on Hot Topics in Networks, 2008.

[15] F. Jiang, S. He, P. Cheng, and J. Chen, "On optimal scheduling in wireless rechargeable sensor networks for stochastic event capture," in MASS, 2011. 

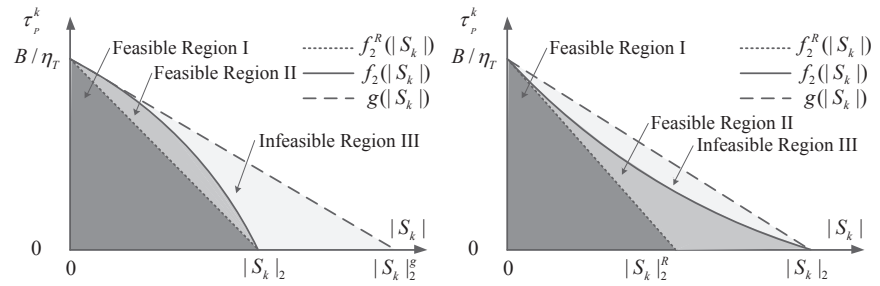

Fig. 8. Feasible Region Analysis Fig. 9. Feasible Region Analysis for the Case where $\eta_{C}<\eta_{T}$ in for the Case where $\eta_{C}>\eta_{T}$ in MinMCP-R

MinMCP-R

[16] V. Nagarajan and R. Ravi, "Approximation algorithms for distance constrained vehicle routing problems," Networks, vol. 59, no. 2, pp. 209-214, 2012.

[17] T. G. Hinton, "A thesis regarding the vehicle routing problem including a range of novel techniques for its solution," Master's thesis, University of Bristol, 2010.

\section{APPENDIX}

\section{A. Proof of Theorem 4.4}

Proof: First of all, it is easy to verify that $f_{2}(0)=\frac{B}{\eta_{T}}$, $f_{2}\left(\left|S_{k}\right|_{2}\right)=0$ where $\left|S_{k}\right|_{2}=\frac{B}{\lambda \cdot\left(B+\eta_{C} \cdot \tau_{\text {vac }}^{c}\right)}$, and $f_{2}\left(\left|S_{k}\right|\right)$ is a decreasing and concave function given that $\eta_{C}<\eta_{T}$ (we don't have to consider the case where $\left|S_{k}\right| \geq \frac{\eta_{T}}{\lambda \cdot\left(\eta_{T}-\eta_{C}\right)}$, since $\left|S_{k}\right|<\frac{1}{\lambda}$ is required for a feasible solution in MinMCP), as illustrated in Fig. 8. Also in Fig. 8, the line $f_{2}^{R}\left(\left|S_{k}\right|\right)$, one of the outputs of Algorithm 1, connects the two points of $f_{2}\left(\left|S_{k}\right|\right)$ at $\left|S_{k}\right|=0$ and $\left|S_{k}\right|=\left|S_{k}\right|_{2}$. The other line, $g\left(\left|S_{k}\right|\right)$, is tangent to $f_{2}\left(\left|S_{k}\right|\right)$ at point $\left|S_{k}\right|=0$, and intersects the horizontal axis at point $\left|S_{k}\right|_{2}^{g}=\frac{B}{\lambda \cdot\left(B \cdot \frac{\eta_{C}}{\eta_{T}}+\eta_{C} \cdot \tau_{\text {vac }}^{c}\right)}$.

A region in the first quadrant is said to be feasible if any point $\tau_{P}^{k}$ within it satisfies the constraint $f_{2}\left(\left|S_{k}\right|\right)$. In Fig. 8 , both of the region I and the region II are feasible, while the region III is not. Denote by $\mathcal{M}^{R *}, \mathcal{M}^{*}$ and $\mathcal{M}^{g *}$ the optimal sets of tours with minimum cardinality constrained by $f_{2}^{R}\left(\left|S_{k}\right|\right), f_{2}\left(\left|S_{k}\right|\right)$ and $g\left(\left|S_{k}\right|\right)$, respectively. Clearly, we have:

$$
\left|\mathcal{M}^{g *}\right| \leq\left|\mathcal{M}^{*}\right| \leq\left|\mathcal{M}^{R *}\right| \text {. }
$$

Using similar analysis in the proof of Theorem 4.3, we know the output of Algorithm 1, $\mathcal{M}$, is subject to:

$$
|\mathcal{M}| \leq 6 \cdot\left(\left\lceil\log _{2} \frac{B / \eta_{T}}{B / \eta_{T}-2 \Delta\left(\frac{\lambda \cdot\left(B+\eta_{C} \cdot \tau_{\text {vac }}^{c}\right)}{\eta_{T}}\right)+2}\right\rceil+1\right) \cdot\left|\mathcal{M}^{R *}\right| \text {. }
$$

Next, we show how to construct a feasible solution $\mathcal{M}^{\prime}$ meeting constraint $f_{2}^{R}\left(\left|S_{k}\right|\right)$ from $\left|\mathcal{M}^{g *}\right|$. In general, we use a partition method to achieve this goal. Suppose there is a tour $P_{k^{r}} \in \mathcal{M}^{g *}$ traversing a set of nodes $\left|S_{k^{r}}\right|$ with travel time $\tau_{P}^{k^{r}}$ located in the region I, II or III, namely:

$$
\tau_{P}^{k^{r}} \leq \frac{B}{\eta_{T}}-\frac{B / \eta_{T}}{\left|S_{k}\right|_{2}^{g}}\left|S_{k^{r}}\right|
$$

We then greedily partition $P_{k^{r}}$ into as few paths as possible, such that each path contains at most $\left\lfloor\frac{\left|S_{k}\right|_{2}}{\left|S_{k}\right|_{2}^{g}} \cdot\left|S_{k^{r}}\right|\right\rfloor$ nodes (apparently, no operation is needed when $\tau_{P}^{k^{r}}$ is located in region I). Subsequently, for each obtained path, we connect its endpoints to the BS in order to form a new tour. Note that the travel time of each newly constructed tour is definitely

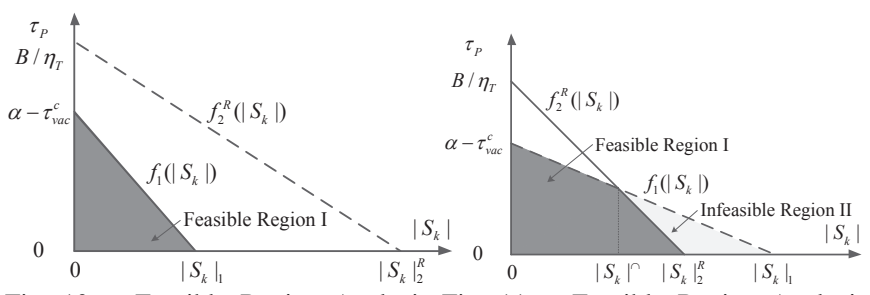

Fig. 10. Feasible Region Analysis Fig. 11. Feasible Region Analysis for the Case where $\alpha-\tau_{v a c}^{c} \leq \frac{B}{\eta_{T}}$ for the Case where $\alpha-\tau_{v a c}^{c}<\frac{B}{\eta_{T}}$ and $\left|S_{k}\right|_{1} \leq\left|S_{k}\right|_{2}^{R}$ in MinMCP and $\left|S_{k}\right|_{1}>\left|S_{k}\right|_{2}^{R}$ in MinMCP

no more than that of $P_{k^{r}}$, i.e., $\tau_{P}^{k^{r}}$, as the weight space is metric. To summarize, for any obtained tour $P_{k_{j}^{r}}$, we have $\left|S_{k_{j}^{r}}\right| \leq\left\lfloor\frac{\left|S_{k}\right|_{2}}{\left|S_{k}\right|_{2}^{g}} \cdot\left|S_{k^{r}}\right|\right\rfloor$ and $\tau_{P}^{k_{j}^{r}} \leq \tau_{P}^{k^{r}}$, Hence:

$$
\begin{aligned}
\tau_{P}^{k_{j}^{r}} & \leq \tau_{P}^{k^{r}} \leq \frac{B}{\eta_{T}}-\frac{B / \eta_{T}}{\left|S_{k}\right|_{2}^{g}}\left|S_{k^{r}}\right| \leq \frac{B}{\eta_{T}}-\frac{B / \eta_{T}}{\left|S_{k}\right|_{2}^{g}} \frac{\left|S_{k}\right|_{2}^{g}}{\left|S_{k}\right|_{2}}\left|S_{k_{j}^{r}}\right| \\
& \leq \frac{B}{\eta_{T}}-\frac{B / \eta_{T}}{\left|S_{k}\right|_{2}}\left|S_{k_{j}^{r}}\right| .
\end{aligned}
$$

Therefore we conclude that $\tau_{P}^{k_{j}^{r}}$ must belong to the region I. In this way, $\left|\mathcal{M}^{g *}\right|$ tours can be finally converted into $\left|\mathcal{M}^{\prime}\right|$ tours meeting constraint $f_{2}^{R}\left(\left|S_{k}\right|\right)$, and is subject to:

$$
\begin{aligned}
\left|\mathcal{M}^{\prime}\right| & \leq\left\lceil\frac{\left|S_{k^{r}}\right|}{\left\lfloor\frac{\left|S_{k}\right|_{2}}{\left|S_{k}\right|_{2}^{g}} \cdot\left|S_{k^{r}}\right|\right\rfloor}\right\rceil \cdot\left|\mathcal{M}^{g *}\right| \\
& \leq\left(2 \cdot \frac{\left|S_{k}\right|_{2}^{g}}{\left|S_{k}\right|_{2}}+1\right) \cdot\left|\mathcal{M}^{g *}\right| \\
& \leq\left(2 \cdot \frac{B+\eta_{C} \cdot \tau_{\text {vac }}^{c}}{B \cdot \frac{\eta_{C}}{\eta_{T}}+\eta_{C} \cdot \tau_{\text {vac }}^{c}}+1\right) \cdot\left|\mathcal{M}^{*}\right|
\end{aligned}
$$

Note the last inequality is obtained by following Equ. (11). Each tour in $\left|\mathcal{M}^{\prime}\right|$ has a travel time feasible for region I. Besides, it is clear that:

$$
\left|\mathcal{M}^{R *}\right| \leq\left|\mathcal{M}^{\prime}\right|
$$

since $\mathcal{M}^{R *}$ is optimal.

Combining Eqs. (12)(15)(16) will give the result.

\section{B. Proof of Theorem 4.5}

Proof: The proof is similar to that of Theorem 4.4, we omit it to save space. Note that for this case, $f_{2}\left(\left|S_{k}\right|\right)$ is convex as shown in Fig. 9. $f_{2}^{R}\left(\left|S_{k}\right|\right)$ is the line tangent to $f_{2}\left(\left|S_{k}\right|\right)$ at point $\left|S_{k}\right|=0$, and $g\left(\left|S_{k}\right|\right)$ is the line connects the two end points $\left|S_{k}\right|=0$ and $\left|S_{k}\right|=\left|S_{k}\right|_{2}$ of $f_{2}\left(\left|S_{k}\right|\right)$.

\section{Proof of Theorem 4.8}

Proof: We only sketch our proof here to save space. After Step 6, the travel time of an obtained tour $k\left(k \in\left[\left|\mathcal{M}^{\prime}\right|\right]\right), \tau_{P}^{k}$, falls in the region I or II, as illustrated in Fig. 11. If $\left|S_{k}\right|>$ $\left|S_{k}\right|^{\cap}, \tau_{P}^{k}$ may fall in the region II, which is infeasible for $f_{2}^{R}\left(\left|S_{x}\right|\right)$. Therefore in Step 9, we use the partition technique proposed in the proof of Theorem 4.4 for tour $k$ to address this problem. In the worst case, the number of newly created

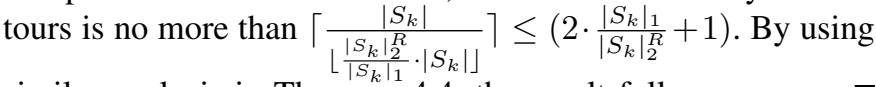
similar analysis in Theorem 4.4, the result follows. 